\title{
Image-Guided Surgery: A Combined Evolution of Surgery and Imaging Methods
}

\author{
Carlos Federico D* and Josefina Cuneo
}

Instituto de Cardiología de Rosario, Argentina

Abstract

Background: From its beginnings to the present, surgical procedures have been improving with the

ISSN : 2688-836X

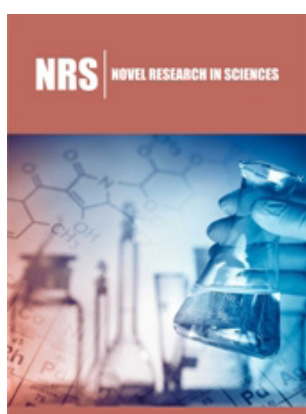

*Corresponding author: Carlos Federico D, Instituto de Cardiología de Rosario, Argentina

Submission: 侮 May 15, 2019

Published: 柴July 11, 2019

Volume 1 - Issue 2

How to cite this article: Carlos F D, Josefina C, Image-Guided Surgery: A Combined Evolution of Surgery and Imaging Methods. Nov Res Sci.1(2). NRS.000506.2019. DOI: 10.31031/NRS.2019.1.000506

Copyright@ Carlos Federico D, This article is distributed under the terms of the Creative Commons Attribution 4.0 International License, which permits unrestricted use and redistribution provided that the original author and source are credited. passage of time. The benefits of modern surgery are remarkable. But to achieve this, several factors were combined, the evolution of surgery and of diagnostic imaging methods. All these factors combined to play a fundamental role in the innovation of image-guided surgery.

Conclusion: Image-guided surgery comprises a field of minimally invasive surgery in which the procedures are carried out through the orientation of the imaging systems. The combined evolution of surgery and imaging methods led to image-guided surgery. Its use is practically daily and its benefits exceptional.

Keywords: Surgery; Image-guided surgery; Minimally invasive surgery

\section{Introduction}

The evolution of science never stopped. The same happens with surgery. From its beginnings to the present, surgical procedures have been improving with the passage of time. They went from bloody and painful operations to painless procedures with minimally invasive approaches [1]. The benefits of modern surgery are remarkable. It can reduce hemorrhagic complications, pain, infections, scars and operative times. But to achieve this, several factors were combined. First, it must be considered that the evolution of surgery as such was continuous. Then came the development of diagnostic imaging methods [2] and invasive direct vision studies. Finally, all these factors combined to play a fundamental role in the innovation of image-guided surgery, which is the latest expression of minimally invasive surgery.

\section{Evolution of image-guided surgery}

The study of the evolution of surgery, the methods of imaging studies and endoscopy will allow us to understand the current meaning of image-guided surgery.

Surgery: The origin of surgery as a discipline goes back to the beginning of civilized society. In the first place, the barbers were the ones who carried out procedures of this type. With the passage of time, the characters that allowed us to give a theoretical framework to this activity stood out. In 1543, the Belgian Andries van Wesel (Andreas Vesalius in Latin, 15141564) published his treatise on anatomy [3]. For this reason, he is considered the father of modern anatomy. While a couple of years later, in 1545, the French physician Ambroise Paré (1510-1590), the father of current surgery [4], published Anatomie Universelle du Corps Humain. One of the main milestones that marked a turning point in the history of surgery was the development of anesthesia and antisepsis. In 1846, Thomas G Morton (1819-1868) applied diethyl-ether for the first time, successfully reducing the pain of a patient undergoing surgery. Before that, the dentist Horace Wells (1815-1848) had tested the use of nitrous oxide with good results [5]. The development of the antisepsis was a little longer. In 1590, Jansen (1585-1632) invented the microscope, a crucial element for the discovery of pathogenic microorganisms. The French physician Louis Pasteur (1822-1895) presented the germinal theory of infectious diseases and pasteurization in 1864. Then, in 1865, the English surgeon Joseph Lister (1827-1912) suspected that surgical infections had a bacterial origin, then who 
developed the antisepsis technique [6]. Continuing in this field, but later in time, there was a great discovery that further impelled the development of surgery. In 1928, Alexander Fleming (1881-1955) discovered that a fungus called Penicillium notatum had grown in a culture of Staphylococcus aureus, thus creating the first antibiotic [7]. All these events were evolutionary and sequential so that one improved the previous one and allowed the next one to develop. The surgical academies were formed with their great teachers. The procedures were standardized; they had an indication and a precise technique. Then came the place of technological advances that we know today. The operating rooms, their lighting, the anesthesia equipment, the electrocautery elements, and the video systems were improved, giving way to the next generation of surgeons.

Radiology: X-rays comprise ionizing electromagnetic radiation. Its application to medicine began in 1895, when the German Wilhelm Röntgen (1845-1923) used x-rays to see the bones of his hand [8]. The great benefits that he presented led to the use of fluoroscopy in a massive way. After knowing the negative effects of radiation, in the 1930s the first means of protection were implemented. With the passage of time, the definitions of fluoroscopic images improved, which allowed interventions with this technology. To achieve this, the Swedish Sven Ivar Seldinger (1921-1998) developed a technique for the introduction of an angiographic catheter in 1953 [9]. The idea of performing procedures on organs using this technology emerged. In 1955, Dr. Goodwin (1915-1998) performed the first percutaneous nephrostomy guided by fluoroscopy [10]. From the systematic application of Seldinger's technique, he began to study central and peripheral circulation. The lesions were discovered in different arteries, such as the coronary arteries. In 1964, Dotter (1920-1985) and Judkins (1922-1985) introduced and described per- cutaneous transluminal angioplasty [9]. The subsequent dissemination of this discipline resulted in vascular and non-vascular interventional radiology being perfectly defined subspecialties.

Endoscopy: The first indications of the use of instruments to observe the interior of the human body date from the sixteenth and seventeenth centuries. It was in 1806 and in 1853 that the development of these devices began, by Bozzini (1773-1809) and Desormeaux (1815-1894), respectively. They used lighting to improve their visualization. The latter was the pioneer in calling this novel device "endoscope" [11]. In 1868, Adolf Kussmaul (18221902) used a precarious gastroscope. Later years, several scholars tried to optimize its use, but the illumination was poor that limited the vision. The German Maximilian Carl-Friedrich Nitze (18481906), spread the use of the first cystoscope in 1877, based on a series of lenses that magnified the vision. This model inspired Jan Mikulicz-Radecki (1850-1905) to invent the first gastroscope [12]. The continuous development improved the illumination of the instrument. Then an air channel was created to lighten the optical lens. The massive use of this device came from the hand of Rudolf Schindler (1888-1968). Despite the innovative nature of endoscopy, it presented great complications. The rigidity of the instrument was associated with esophageal perforations. Over time, semi-flexible endos- copes were developed, reaching the flexible fiberscope that we know today, thanks to Prof. Basil Hirschowitz (1925-2013) in 1957 [11]. The improvement in the video system was applied to the endoscopy industry, which resulted in a video endoscopy. From here, endoscopy progressed by leaps and bounds. They improved the visualization systems and the definition of the images. In this way, the diagnostic processes were more precise and conditions for the next stage, endoscopic surgical procedures, were given. In 1968, Dr. William S. McCune (1909-1998) performed the first endoscopic retrograde cholangiopancreatography (ERCP) [12]. Years earlier, other physicians injected contrast into the bile and pancreatic ducts, opacifying the ducts by fluoroscopy. Procedures continued, challenging the techniques of the time. In the 1970s, electrocautery was applied in endoscopy, developing the first endoscopic polypectomies and the control of digestive hemorrhages, both gastric and colonic. The invention of different devices allowed to perform other procedures, such as biopsies, dilations, and placement of prostheses. In this last time, 2 studies were combined by images for a common objective, as was the case with ERCP. The endoscopy application together with the ultrasound formed the echoendoscope. Fundamental artifact to detect lesions in organs near the esophagus and the stomach, in addition to being used as a guide for the puncture of the same [13]. As we can see, the current utility of endoscopy stopped being exclusively diagnostic to become an interventionist. This attracted the attention of many surgeons who innovated the combined procedures. This is how the NOTES technique took its first steps in recent years, achieving a trans gastric cholecystectomy in an animal model [14]. Although there are several articles published on this approach, it still requires further development. It could represent the next step in the evolution of minimally invasive surgery [15].

Ultrasonography: Ultrasonography is a safe, economical and operator-dependent method. It is one of the methods of diagnostic by older images. His starting point was in the 1940s. American professor George Ludwig was one of the first to use ultrasound in medicine. In 1942, Dr. Karl T. Dussik (1908-1968) sought to detect brain tumor lesions using what he called "hyper phonography." It consisted of passing a sonic ray through the skull while measuring its attenuation of the ultrasound [16]. Dr. Douglas Howry continued to investigate this new technology until in 1953 he published twodimensional images of the forearm through ultrasonography. It was in 1951 when the English surgeon John JWild (1914-2009) published images of tumor lesions, which makes this method of diagnostic importance [17]. A year later, he published an article about the anatomical cut of the forearm in real time, using ultrasonography. He did this work with the electronic engineer John Reid. With the passage of time, this system was perfected. The linear mode for the study of the structures and the Doppler effect to analyze the flows and their speeds appeared. The gray scale and colors were added, which facilitates the interpretation of what is visualized by the operator [18]. Its application became massive in surgery, gynecology, obstetrics, and cardiology. At present, the systems 
by images improved remarkably. The ultrasound was developed in 3D and 4D. It allowed detecting pre and postnatal structural pathologies with great precision. In addition, the definition of the images improved with the use of contrast to differentiate the characteristics of the tissues studied [19]. Ultrasonography is very useful in percutaneous procedures. This is because it is a method that can be used in real time and next to the patient's bed, unlike tomography. In addition, it does not bring with it the complications of radiation associated with fluoroscopy. Many invasive procedures, such as biopsies, drainages, puncture aspirations and even tumor ablations are guided by ultrasonography.

Computed tomography: The discovery of X-rays and their application to medicine allowed us to observe the interior of the human body without the need for surgery. The diagnoses are established by contrasting the densities of different tissues after X-rays pass through the body. Despite this, this method has certain limitations. It was not possible to detect lesions in organs with similar density. This problem was solved by the invention of computed tomography (CT). This was in 1971 by engineer Godfrey Hounsfield (1919-2004) [20]. The novel device was used for the first time in the Atkinson Morley Hospital for a brain scan. It presented a rapid evolution in the following years. The resolution of the images was improved obtaining thinner cuts and the time required for the study was optimized [21]. This allowed full body scans after a few years. We always try to improve the quality of the images obtained. The introduction of contrast material, both oral and intravenous, facilitated the interpretation of intraparenchymal and intraluminal lesions. Since the $90 \mathrm{~s}$, CT was already a method of studying images disseminated worldwide. The main medical centers had one of this equipment. Radiologists saw that CT was not just a diagnostic tool. The preoperative images of the tumor lesions observed in the liver guided the surgeon when performing an open surgical biopsy. With the passage of time, it was demonstrated that it was possible to perform liver biopsies, practically in real time, using the images obtained through the CT-guided. The industry of materials for percutaneous use, such as catheters, wire guides, and needles developed rapidly. They went from being the exclusive spring of endoscopic procedures to sharing prominence with percutaneous interventions. These evolutions in parallel allowed that currently many procedures stopped being purely surgical and turned into minimally invasive surgeries guided by images, such as biopsy punctures, drainage collections, tumor ablations, among others [22].

Magnetic resonance imaging: The origins of Nuclear Magnetic Resonance (MR) take place in studies conducted by Isidor Isaac Rabi (1898-1988) in 1938, and by Felix Bloch (1905-1983) and Edward Mills Purcell (1912-1997) later, in 1952. He studied that by sending a beam of molecules through a magnetic field, radio waves could be obtained. In 1971, Raymond Damadian (1936) proposed using MR to differentiate between healthy tissue and cancer. $\mathrm{He}$ was the first creator of an MR machine. The development of this system continued in the hands of Peter Mansfield (1933-2017) and Paul Lauterbur (1929-2007). The latter showed, in 1973, that it was possible to obtain MR images. Four years later, in 1977, the first images of human Magnetic Resonance Imaging (MRI) were published [23]. The evolution of this system was observed, above all, in the characteristics of its images. Currently, elastography, spectrograph, 3D reconstructions, enhanced images with contrast, among others, can be obtained from a simple MRI-scan. One of the main limitations of the application of MRI in the image-guided procedures is that it is not possible to use metallic instruments, such as needles, embolization coils, etc. Today, this problem has been solved with the development of carbon needles and embolizing coils compatible with the MRI system.

Laparoscopy: Minimally invasive procedures with direct vision always aroused interest in surgeons [24]. The first signs date back to 1950 when Dr. R Palmer (1904-1985) used laparoscopy for the first time to make a diagnosis. The development of this technique, initially not well accepted, was done slowly. Until 1981, Dr. K Semm (1927-2003) performed the first laparoscopic surgical procedure, an appendectomy. Only 6 years later, in 1987, Dr. P Mouret (19382008) opened the doors to some of the procedures most commonly performed today, carrying out the first laparoscopic cholecystectomy $[2,25]$. This surgery, although resisted in its beginnings, was quickly learned by modern surgeons, and had to be accepted by the most traditional surgeons. It had a rapid diffusion. Its main weakness, the surgical lesion of the bile duct, was reduced to a minimum when polishing the technique [26]. Little by little, the surgeons were encouraged to perform increasingly challenging procedures. Nowadays, a hernioplasty, colectomy, or laparoscopic gastrectomy, among other procedures, are commonplace [27]. As expected, the imaging methods applied to the surgery were not slow to move to laparoscopy. Intraoperative cholangiography was obtained with contrast injection to the biliary tree and fluoroscopy. This facilitated the detection of choledocholithiasis and its treatment in a single surgery [28]. Soon the laparoscopic ultrasound transducer was developed. It made it possible to perform an ultrasonographic scan of the liver or pancreas during the operative act. It facilitated the localization of tumors and their relationship with vascular structures. Occasionally, it may clarify the biliary anatomy during cholecystectomy [29]. These last few years have shown that not only imaging methods can be applied to surgery. Different surgical techniques can be combined with each other. Laparoscopy may be associated with percutaneous surgery. Thus, procedures such as punctures of liver biopsies can be carried out during a laparoscopic cholecystectomy. The exact delineation of a colorectal tumor during a laparoscopic colectomy can be determined by an intraoperative colonoscopy [30]. The same principle can be applied to a laparoscopic gastrectomy for a gastric tumor [31]. The last twenty years of evolution in medicine, and especially in laparoscopic surgery, laid the foundations for the development of the maximum technology at the service of surgical interventions, robotic surgery. The concept of telepresence is represented in this way [32]. Different companies financed the manufacture of robots. From this moment several procedures were carried out using the robotic system [33]. The procedure that marked a milestone in the 
history of robotic surgery was the so-called "Operation Lindbergh". It consisted of a transcontinental robot-assisted laparoscopic cholecystectomy. The patient was in Strasbourg (France) while the surgeon operated from New York (USA) [34]. As at the beginning of laparoscopy, robotic surgery found detractors but then developed rapidly in the world's leading centers.

Image-guided surgery: Image-guided surgery is a new concept. It comprises a field of minimally invasive surgery in which the procedures are carried out through the orientation of the imaging systems. The methods of diagnostic imaging have developed a lot in the last time. They perform their task faster and present a better definition than previous years. Evolution in this field influenced the growth and massive use of image-guided procedures [35]. Such is the case that, currently, it is considered as the first option of approach to performing percutaneous biopsy punctures, collection drains, and tumor ablations. The surgery and its related procedures have changed markedly. Some years ago, almost all cases in which patients had lower limb ischemia secondary to arterial stenosis were resolved with surgery. After the development of interventional radiology, this problem began to be re- solved percutaneously, by balloon dilation and placement of prostheses. In this way, the surgery was replaced by a minimally invasive approach. Over the years and the familiarization of surgeons with the diagnostic imaging systems allowed to innovate in non-vascular intervention techniques. The catheters that were used in the drainage of the bile duct or in neph rostomies were adapted. Then, first, the ultrasound and the tomography allowed later to observe lesions in solid organs, establishing diagnostic imaging. The confirmation of the pathology had to be done obtaining a surgical biopsy of the tumor. Later, the imaging systems served as a guide for the punctures to perform biopsies. Once again, the surgery was replaced by a minimally invasive method. The surgery was preserved for the treatment of the tumor. But these last years witnessed the appearance of different tumor ablation systems. These, guided by images, allowed the treatment of tumors in different organs. The image-guided procedures stopped being only diagnoses and became therapeutic. The maximum expression of the systems of images at the service of surgery are the hybrid operating rooms [35]. They consist of an operating table surrounded by a CT equipment, an ultrasound on the bed and a fluoroscopy system. They can be used before, during or immediately after surgery. It allows reducing the transfer time between the operating room and the medical imaging room, as well as facilitating the completion of complex procedures. The reconstruction of 3D images allows locating intraoperative tumors. The injection of contrast into the vascular or biliary tree facilitates the surgical planning of both resection (for example liver tumor resection) and reconstructive surgery (for example biliodigestive anastomosis), almost in real time. Image-guided surgery is part of the current therapeutic armamentarium. It is used by most cuttingedge centers around the world. We know that it is not the final stage of evolution. It is just one more link in the continuous development of this discipline, which stopped being individual to become multidisciplinary. Includes surgeons, radiologists, endoscopists, among others. Very soon the surgeon of the future must have a broad theoretical and practical know- ledge about endoscopy, radiology, laparoscopy. And maybe, soon, in Artificial Intelligence (AI), Augmented Reality (AR) and robotics, since the combination of these factors seems to be the next qualitative leap in surgery.

\section{Conclusion}

Image-guided surgery comprises a field of minimally invasive surgery in which the procedures are carried out through the orientation of the imaging systems. The combined evolution of surgery and imaging methods led to image-guided surgery. This new discipline is present in most centers. Its use is practically daily and its benefits exceptional. Conflict of Interest: All authors declare to have no conflict of interest.

\section{References}

1. Gawande A (2012) Two hundred years of surgery. N Engl J Med 366(18): $1716-1723$.

2. Villet $\mathrm{R}$ (2011) From ambroise paré to the surgeon of the $21^{\text {st }}$ century, or, from surgical ligation to robots. J Visc Surg 148(5): e1-2.

3. Zampieri F, Maghawry M, Zanatta A, Thiene G (2015) Andreas vesalius: celebrating 500 years of dissecting nature. Glob Cardiol Sci Pract 22(5): 66.

4. Hernigou P (2013) Ambroise paré's life (1510-1590): part I. Int Orthop 37(3): 543-547.

5. Menczer LF, Jacobsohn PH (19912) Dr horace wells: the discoverer of general anesthesia. J Oral Maxillofac Surg 50(5): 506-509.

6. Brand RA (2010) Biographical sketch: baron joseph lister, FRCS, 18271912. Clin Orthop Relat Res 468(8): 2009-2011.

7. Kyle RA, Shampo MA (1979) Sir alexander fleming. JAMA 241(17): 1781.

8. Scatliff JH, Morris PJ (2014) From röntgen to magnetic resonance imaging: the history of medical imaging. NC Med J 75(2): 111-113.

9. Baum RA, Baum S (2014) Interventional radiology: a half century of innovation. Radiology 273(2): S75-91.

10. Patel SR, Nakada SY (2015) The modern history and evolution of percutaneous nephrolithotomy. J Endourol 29(2): 153-157.

11. Goh KL (2015) $24^{\text {th }}$ seah cheng siang lecture: seeing better, doing better evolution and application of gastrointestinal (GI) endoscopy. Ann Acad Med Singapore 44(1): 34- 39.

12. Morgenthal CB, Richards WO, Dunkin BJ, Forde KA, Vitale G, et al. (2007) The role of the surgeon in the evolution of flexible endoscopy. Surg Endosc 21(6): 838-853.

13. Cazacu IM, Luzuriaga CAA, Saftoiu A, Vilmann P, Bhutani MS (2018) A quarter century of EUS-FNA: Progress, milestones, and future directions. Endosc Ultrasound 7(3): 141-160.

14. Perretta S, Dallemagne B, Coumaros D (2008) Natural orifice transluminal endoscopic surgery: transgastric cholecystectomy in a survival porcine model. Surg Endosc 22: 1126-1130.

15. Sugimoto M (2009) Natural orifice translumenal endoscopic surgery (NOTES) for innovation in hepatobiliary and pancreatic surgery: preface. J Hepatobiliary Pancreat Surg 16(3): 247-248.

16. Shampo MA, Kyle RA (1995) Karl the odore dussik pioneer in ultrasonography. Mayo Clin Proc 70(12): 1136.

17. Shampo MA, Kyle RA (1997) John julian wild--pioneer in ultrasonography. Mayo Clin Proc 72(3): 234.

18. Claudon M, Tranquart F, Evans DH, Lefèvre F, Correas M (2002) Advances in ultrasound. Eur Radiol 12(1): 7-18. 
19. Cosgrove DO (2017) Evolution of ultrasonography since the 1970s. Ultrasound Med Biol 43(11): 2741-2742.

20. Beckmann EC (2006) CT scanning the early days. Br J Radiol 79(937): 5-8.

21. Rubin GD (2014) Computed tomography: revolutionizing the practice of medicine for 40 years. Radiology 273(2): S45-74.

22. Soares GM, Murphy TP (2005) Clinical interventional radiology: parallels with the evolution of general surgery. Semin Intervent Radiol 22(1): 10-14.

23. Edelman RR (2014) The history of MR imaging as seen through the pages of radiology. Radiology 273(2): S181-200.

24. Radojcić B, Jokić R, Grebeldinger S, Meljnikov I, Radojić N (2009) History of minimally invasive surgery. Med Pregl 62(11-12): 597-602.

25. Spaner SJ, Warnock GL (1997) A brief history of endoscopy, laparoscopy, and laparoscopic surgery. J Laparoendosc Adv Surg Tech A 7(6): 369373.

26. Sajid MS, Leaver C, Haider Z, Worthington T, Karanjia N, et al. (2012) Routine on-table cholangiography during cholecys- tectomy: a systematic review. Ann R Coll Surg Engl 94(6): 375-380.

27. Himal HS (2002) Minimally invasive (laparoscopic) surgery. Surg Endosc 16(12): 1647-1652.
28. Ford JA, Soop M, Du J, Loveday BP, Rodgers M (2012) Systematic review of intraoperative cholangiography in cholecystectomy. Br J Surg 99(2): 160-167.

29. Dili A, Bertrand C (2017) Laparoscopic ultrasonography as an alternative to intraoperative cholangiography during laparoscopic cholecystectomy. World J Gastroenterol 23(29): 5438-5450.

30. Gorgun IE, Aytac E, Manilich E, Church JM, Remzi FH (2013) Intraoperative colonoscopy does not worsen the outcomes of laparoscopic colorectal surgery: a case-matched study. Surg Endosc 27(10): 3572-3576.

31. Xuan Y, Hur H, Byun CS, Han SU, Cho YK (2013) Efficacy of intraoperative gastroscopy for tumor localization in totally laparoscopic distal gastrectomy for cancer in the middle third of the stomach. Surg Endosc 27(11): 4364-4370.

32. Pugin F, Bucher P, Morel P (2011) History of robotic surgery: from AESOP $®$ and ZEUS $®$ to da Vinci®. J Visc Surg 148(5): e3-8.

33. Lane T (2018) A short history of robotic surgery. Ann R Coll Surg Engl $100(6): 5-7$.

34. Marescaux J, Leroy J, Gagner M, Rubino F, Mutter D, et al. (2001) Transatlantic robot-assisted telesurgery. Nature 413(6854): 379-380.

35. Linte CA, Yaniv ZR (2016) Image-guided interventions: we've come a long way, but are we there? IEEE Pulse 7(6): 46-50.

For possible submissions Click below: 